\title{
Presencia, utilización y aprovechamiento de las TIC en la formación académica estudiantil
}

\author{
Luis-Alberto Paz-Pérez, Gerardo Tamez-González, Abraham Hernández-Paz y \\ Oswaldo Leyva-Cordero
}

\section{RESUMEN}

El presente trabajo empírico se desarrolla desde la perspectiva de la comunidad estudiantil en cuanto a la existencia y utilización práctica de las tecnologías de la información y comunicación (TIC) en el ámbito de las tareas académicas cotidianas de la Facultad de Ciencias Políticas y Administración Pública de la Universidad Autónoma de Nuevo León. La propuesta tiene como objetivo describir la percepción de los alumnos sobre las TIC y su grado de aplicación en su formación académica. El proyecto es de tipo cuantitativo, transversal, desarrollado a través de un cuestionario aplicado a una muestra estratificada por semestre. Los hallazgos detectados durante el proceso y los resultados obtenidos, permiten develar la existencia de equipamiento suficiente y adecuado, con lo cual se concluye que existen las condiciones óptimas para ampliar la oferta educativa y ofrecer programas formativos totalmente a distancia, utilizando las TIC.

Palabras clave: tecnologías, TIC, educación virtual, educación a distancia, México.

Luis-Alberto Paz-Pérez

Mexicano. Doctor en Educación por la Escuela Normal de Sinaloa, México. Docente de Tiempo Completo y Jefe del Departamento de TIC en la Facultad de Ciencias Políticas y Administración Pública en la Universidad Autónoma de Nuevo León, México. Temas de investigación: gestión y política educativa.

\section{Gerardo Tamez-González}

gerardo.tamezg@uanl.mx

Mexicano. Doctor en Gerencia y Política Educativa por el Centro de Estudios Universitarios de Baja California, México. Docente de Tiempo Completo y Director de la Facultad de Ciencias Políticas y Administración Pública en la Universidad Autónoma de Nuevo León, México. Temas de investigación: gestión y política educativa.

\section{Abraham Hernández-Paz}

abraham.hernandezp@uanl.mx Mexicano. Doctor en Gerencia y Política Educativa por el Centro de Estudios Universitarios de Baja California, México. Docente de Tiempo Completo y Subdirector General de la Facultad de Ciencias Políticas y Administración Pública, Universidad Autónoma de Nuevo León, México. Temas de investigación: gestión y política educativa. 


\section{Presença, utilização e aproveitamento das TIC na formação acadêmica estudantil}

\section{RESUMO}

O presente trabalho empírico se desenvolve desde a perspectiva da comunidade estudantil em quanto à existência e utilização prática das tecnologias da informação e comunicações (TIC) no âmbito das tarefas académicas cotidianas da Facultad de Ciencias Políticas y Administración Pública da Universidad Autónoma de Nuevo León. A proposta tem como objetivo descrever a percepção dos alunos sobre as TIC e seu grau de aplicação em sua formação académica. O projeto é de tipo quantitativo, transversal, desenvolvido através de um questionário aplicado a uma amostra estratificada por semestre. Os descobrimentos detectados durante o processo e os resultados obtidos, permitem desvelar a existência de equipamento suficiente e adequado, com o qual se conclui que existem as melhores condições para ampliar a oferta educativa e oferecer programas formativos totalmente a distância, utilizando as TIC.

Palavras chave: tecnologias, TIC, educação virtual, educação a distância, México.

\section{Presence, use and exploitation of ICT in student academic training}

\section{ABSTRACT}

This empirical work is developed from the perspective of the student community regarding the existence and practical use of information and communication technologies (ICT) in the daily academic tasks of the School of Politic Science and Public Administration of the Autonomous University of Nuevo León. The aim of the proposal is to describe the students' perception about ICT and the extent to which they make use of them in their academic training. The project is of a quantitative transversal type, developed through a questionnaire applied to a stratified sample of students per semester. The findings that were made during the process and the results reveal the existence of sufficient and adequate equipment, which leads to the conclusion that the optimum conditions exist to expand the educational offer and offer wholly distance learning programs using ICT.

Key words: technologies, ICT, virtual education, distance education, Mexico. 


\section{Introducción}

El proceso educativo ha sido un ente vibrante y activo desde la aparición del hombre y a través del tiempo, este proceso se va adaptando al entorno social, político y económico al que obedece. Sin menoscabo de las acciones que cada institución educativa pretenda al interior de sus currículos, el proceso se perfecciona cada vez más en una dinámica sin precedente, actualmente, producto del uso de la tecnología, la cual envuelve a la educación así como al resto de las áreas del conocimiento. Sucede con tanta rapidez que a veces no se digiere del todo, por ejemplo, podemos decir que en tan sólo 130 años se ha pasado de la invención del automóvil (1885, Karl Benz, el primer automotor de tres ruedas en Alemania), a los autos eléctricos, los celulares tipo pulsera, los televisores inteligentes, entre otros (Castillejo, 2007).

A partir de los años sesenta se hacen latentes las experiencias que abordan el tema de las tecnologías de la información y las comunicaciones (TIC) en el ámbito educativo. Pero todas ellas convergen en que éstas fueron incluidas en el proceso formativo por la aparición de las mismas en la existencia de la humanidad. Esto habla de que no surgen para cubrir alguna necesidad innata dentro del proceso cognitivo de la enseñanza y aprendizaje, sino para reorientar la praxis docente en la educación, así como los escenarios de trabajo en las empresas y de interacción entre la sociedad.

Según Vidal (2006), los primeros indicios de la incursión de la tecnología en la educación se presentan en 1918, pero es hasta los cincuenta que se desarrollan con mayor intensidad. En la década de los sesenta se manifiesta la revolución electrónica haciéndose evidente en la radio y la televisión, modificando substancialmente la comunicación entre las masas. Y a partir de los setenta se consolida la utilización de las computadoras, como una alternativa de grandes posibilidades en la educación. En los ochenta la integración de las tecnologías en el ámbito educativo se vuelve un tema muy recurrente.
Desde la perspectiva de Sarramona (1990), en los albores de los noventa en el ámbito mundial, la educación se vió inmersa en una dinámica de cambio debido a la irrupción de las TIC, factor determinante que propició realizar los ajustes necesarios en los currículos de las instituciones educativas así como en la forma de interacción con el medio ambiente y la comunicación.

Sin perder relevancia, la inclusión de las tecnologías en las aulas sigue presentándose hoy en día como un tema pertinente en los trabajos de investigación empírica al interior de las instituciones. Como ejemplo, en 2010 la Organización de Estados Iberoamericanos (OEI) publica el documento $\mathrm{Me}^{-}$ tas Educativas 2021 para los países iberoamericanos, donde se establece, entre otras cosas, que la razón de alumnos por computadora debe pasar de un equipo por cada 40 alumnos en 2015 a un equipo por cada 10 alumnos en 2021, y que la frecuencia de uso de los equipos de cómputo por parte de maestros y estudiantes se realice de forma habitual (OEI, 2010).

En respuesta a estas transformaciones, la Universidad Autónoma de Nuevo León (UANL) en su Plan de Desarrollo Institucional (2008), ha generado estrategias que permiten la innovación, y con ello el cambio educativo que propicia la responsabilidad social, equidad e inclusión, conceptos que se manejan actualmente en el ámbito educativo para cubrir el aspecto de la humanización de la educación.

En septiembre de 2008 el Consejo Universitario de la UANL aprueba el Modelo Educativo UANL y surgen una serie de estrategias que han impactado en la reformulación de su currículo. Dicho Modelo privilegia el uso de las tecnologías de la comunicación e información para la promoción de los procesos centrados en el aprendizaje y la formación integral del alumnado (UANL, 2008). Con el fin de conocer los resultados de este entorno tecnológico, es que se elabora el presente proyecto. 


\section{Planteamiento del problema}

En la educación, la forma de transmitir los saberes conlleva la implicación de una modalidad, enfoque o proceso acorde a las necesidades, cultura, saberes profesionales, entendimientos generacionales, hábitos y condiciones de acceso de quien los recibe. Lo que orilla a la reflexión de que en la incorporación de las herramientas de TIC en las actividades humanas (la educación en este caso), se requiere adaptar dichas herramientas a la diversidad social; ya que existen países en desigualdad de oportunidades.

Por otro lado, de acuerdo con Torres (2006), la educación a distancia se ha vinculado históricamente al desarrollo de las tecnologías contemporáneas quedando de manifiesto la utilización de los medios de comunicación presentes en cada época para la formación educativa de las personas; como lo era la utilización del correo físico en su momento y la utilización del correo electrónico hoy en día.

Bajo estas dos premisas, se puede acotar que para proporcionar contenido educativo utilizando las TIC, se debe entender que no existe un modelo único y que cada institución construye su propio modelo; induciendo en ese sentido las premisas de la inclusión social, equidad, cultura, ideología y política educativa.

Por ello, la presente investigación asume el compromiso de esbozar la presencia y el grado de utilización de las TIC en la Facultad de Ciencias Políticas y Administración Pública (FCPyAP) de la UANL por parte de los estudiantes, con el fin de definir y determinar el impacto de las mismas en las actividades formativas de los educandos; además de prospectar la implementación de los programas educativos que se ofertan en la facultad de manera cien por ciento virtual.

En este contexto, la UANL cuenta con dos plataformas (NEXUS y VirtUANL), las cuales soportan actualmente unidades de aprendizaje en forma semipresencial, con ello se establecen las condiciones básicas para la realización del proceso educativo totalmente en línea. Sin embargo, es necesario conocer el aprovechamiento que se tiene de dichas plataformas por parte de los aprendices, aunado a la explotación de estas herramientas para posibilitar la transferencia de conocimiento a distancia utilizando TIC.

Actualmente en el marco de las tecnologías, las opciones de formación que tienen los educandos de la FCPyAP y que se ofertan de forma presencial y semi-presencial son las siguientes:

- Licenciatura en Relaciones Internacionales con acentuaciones en:

- Comercio Exterior y Aduanas

- Gestión y Administración Turística

- Licenciatura en Ciencias Políticas y Administración Pública con acentuaciones en:

- Mercadotecnia Política y Gestión de Medios

- Gestión y Desarrollo Municipal

- Licenciatura en Administración de Energía y Desarrollo Sustentable

En este sentido, cabe destacar la necesidad de ofrecer educación amplia y suficiente a los educandos; la Universidad Autónoma de Nuevo León refrenda su compromiso social con la juventud nuevoleonesa estableciendo flexibilidad en su currículo, fincando para tal efecto modelos que permitan la educación fuera de los confines de las aulas, en horarios acordes a las necesidades de los profesionales y con plataformas tecnológicas que permitan el acceso a los materiales precisos; dando pie a una formación integral y socialmente responsable, que le permita a los aprendices desempeñarse eficientemente como profesionales comprometidos con el desarrollo sustentable, económico, científico, social y cultural, a nivel regional, nacional y mundial (UANL, 2012).

\section{Objetivo}

Determinar el grado en que la comunidad estudiantil percibe en su formación académica la presencia y el aprovechamiento de las TIC en la Facultad de Ciencias Políticas, en su formación académica, 
actividad que permitirá prospectar programas educativos a distancia.

\section{Justificación}

El tema de la educación a distancia es relevante y pertinente debido a la masificación educativa, la cual rebasa la capacidad de infraestructura instalada de las instituciones educativas para atender a un número cada vez mayor de estudiantes. El presente estudio permite visualizar la praxis estudiantil versus el uso de las TIC existentes en la FCPyAP y detectar fortalezas y debilidades, para visualizar la implementación de programas virtuales a distancia.

Se pretende que el estudio se logre, mediante un análisis valorativo, como un documento que establezca las condiciones necesarias para lograr la oferta educativa virtual en la institución. De tal manera que con este tipo de acciones se logre dar cabal cumplimiento a la visión 2020 definida en el Plan de Desarrollo Institucional, mismo que exhorta a flexibilizar la educación y a establecer los pilares para que la UANL se logre como una institución de nivel mundial.

\section{Marco teórico Tecnologías}

Es palpable el cambio que ha sufrido la sociedad en las diferentes actividades que realiza en los ámbitos económico, político e inclusive en el cultural, debido a la inclusión de las tecnologías en las actividades humanas.

Desde la conceptualización de Bunge (citado en Sarramona 1990: 13), "la tecnología es la técnica que emplea conocimientos científicos y se vincula con el concepto clásico del teckné (técnica mecánica), donde Aristóteles distinguía entre el saber hacer simple, con el saber hacer con conocimiento de causa"; y según Colom (citado en Sarramona 1990: 13) "cuando ésta [la técnica] se engarza como objeto de conocimiento (logos), surge entonces la tecno-logía. Misma que se acuña, cuando lo mecánico se pretende resolver mediante la aplicación de técnicas (teóricas); de ahí entonces que se afirma que la tecnología, es la teoría de la técnica"

El desarrollo de la tecnología en los campos que constituyen la vida del hombre moderno es un hecho, y de ello dan cuenta las condiciones en que discurren las tareas cotidianas hoy en día; sin embargo, "se puede postular que debe existir una estrecha relación entre aprendizaje, generación de conocimiento, innovación continua y el uso de las tecnologías" (Cobo, 2009: 298).

Es de dominio público el acelerado crecimiento que han presentado las tecnologías en el último siglo entendiéndose como un progreso social; sin embargo "dicho mejoramiento no se permea completamente en todos los países, debido a lo cual existen brechas entre ciertos sectores que se ven marginados en el uso y aprovechamiento de tales recursos tecnológicos" (Tello, 2008: 3).

En este sentido se han realizado profundos cambios en la ciencia, originando la tercera revolución industrial, cuyas transformaciones son fundamentalmente intelectuales. Dicha evolución se mundializa sentando las bases de una economía basada en la información y se privilegia el conocimiento, el cual desempeña un papel fundamental en las actividades sociales contemporáneas (Tello, 2008).

De acuerdo con Cobo (2009: 298), en la llamada nueva economía "el papel que desempeñan las sociedades del conocimiento es estratégico", en particular las entidades educativas y las organizaciones orientadas a impulsar investigación y desarrollo (I+D), innovación, generación e intercambio de conocimiento y nuevas tecnologías.

De este modo, se procura contribuir desde esas entidades, conocer qué son y para qué sirven dichos instrumentos. Esto último, en la necesidad de desarrollar habilidades vinculadas a la gestión estratégica de la información y el conocimiento (Cobo, 2009). Cobo argumenta que resulta interesante analizar qué se entiende por TIC y conocer y comparar las 
diferentes conceptualizaciones y dimensiones de dichas tecnologías.

\section{Tecnologías de la información y Comunicación (TIC)}

Antes que las computadoras e internet hicieran su aparición, la radio y la televisión ya habían llegado a las escuelas. "A comienzos de la década de los cincuenta, los programas educacionales transmitidos por radio ya eran utilizados en el aula y retransmitidos por maestros prácticamente en todas las localidades de América Latina, Asia y África. Indonesia ha utilizado la radio para llegar a escolares diseminados en sitios aislados" (UNESCO, 2003: 5).

Buscando entender el concepto, se observan las siguientes definiciones:

Las TIC se definen colectivamente como innovaciones en microelectrónica (microprocesadores, semiconductores, fibra óptica, etc.), computación (hardware y software) y telecomunicaciones; las cuales permiten el procesamiento de grandes cantidades de información, almacenamiento y rápida distribución de las mismas a través de redes de comunicación. [...] Herramientas que las personas utilizan para reunir, procesar y compartir información, además de comunicarse entre sí o en grupos por medio de redes computacionales (Fernández, citado en Cobo, 2009: 305).

Nos referimos a ellas como una serie de nuevos medios que van desde hipertextos, multimedios, realidad virtual, internet o televisión digital; de tal manera que su factor común son las redes computacionales que permiten interactuar a dispositivos informáticos inclusive desde sitios remotos. [...] Las nuevas tecnologías se diferencian de los métodos tradicionales de comunicación en las posibilidades de creación de nuevos entornos comunicativos y expresivos que facilitan a los receptores la posibilidad de desarrollar nuevas experiencias formativas, expresivas y educativas (Almenara et al., citado en Cobo, 2009: 305).
Las TIC, son las tecnologías que se necesitan para la gestión y transformación de la información, y muy en particular el uso de ordenadores y programas que permiten crear, modificar, almacenar, proteger y recuperar esa información. [...] Las TIC, como elemento esencial de la sociedad de la información, habilitan la capacidad universal de acceder y contribuir a la información, las ideas y el conocimiento. Hacen, por tanto, posible promover el intercambio y el fortalecimiento de los conocimientos mundiales en favor del desarrollo, permitiendo un acceso equitativo a la información para actividades económicas, sociales, políticas, sanitarias, culturales, educativas y científicas, dando acceso a la información que está en el dominio público (Fundación Telefónica, citado en Cobo, 2009: 306).

De las definiciones anteriores, es interesante acotar la significación en el uso de la electrónica al servicio de la generación, resguardo e intercambio de información y la interacción entre las personas vía remota, puntualizando entre las actividades sociales a la educación.

En este sentido, internet y las TIC están revolucionando la interacción humana, la educación no es la excepción, el formato y la entrega de saberes se han modificado substancialmente. El advenimiento o intrusión de dichos avances han permitido que se modifiquen los paradigmas educativos permitiendo la flexibilización de dicho proceso.

Como apuntan Dussel y Quevedo (2010: 11), "la inclusión de las TIC en los procesos formativos emergen un tanto, por las transformaciones en la economía, política, sociedad y cultura", dado que se requiere que las nuevas generaciones adopten las competencias tecnológicas necesarias en los procesos productivos que les permitan adoptar los nuevos paradigmas en la generación de la riqueza.

Así, las instituciones de educación superior requieren dotar a los espacios áulicos del hardware y software necesarios para la formación de los 
educandos. Con ello cumplen con una exigencia del mercado, mas no tanto con una necesidad educativa como tal. Desde la perspectiva de Fernández (2013), en el proceso cognitivo de la educación el uso de las tecnologías no representa un instrumento que refuerce el proceso mental del aprendizaje, más bien lo complementa.

En otra vertiente, la Organización de las Naciones Unidas para la Educación, la Ciencia y la Cultura (UNESCO) ha señalado que "el acceso universal a la información y a la educación, la libertad de expresión y la diversidad cultural, son los cuatro principios que regirán a las futuras sociedades del conocimiento" (UNESCO, 2003: 5).

Los centros educativos de los países miembros de la Organización para la Cooperación y el Desarrollo Económico (OCDE) tomaron conciencia de esto y los adoptaron de tal manera que del año $2000 \mathrm{al}$ 2012, el promedio de gasto en educación primaria a terciaria de los países en dicho organismo se elevó de 4.8 a $5.2 \%$ del PIB, destacando Nueva Zelanda con el mayor porcentaje $(6.8 \%)$ (OCDE, 2015).

\section{Educación}

"Del latín educare, que significa conducir, guiar, orientar; pero semánticamente recoge la versión de educere que significa hacer salir, extraer, dar a luz" (Picardo, Escobar y Balmore, 2005: 92). Esto orilla a una definición fáctica de que la educación se percibe como un proceso intencional y personal de asimilación cultural, moral y conductual, mediante el cual las personas incorporan el patrimonio cultural de sus antepasados, definiendo con ello su personalidad (Castillejo, 2007); esto puede guiar a una solución ecléctica de que la educación es la intervención y el perfeccionamiento de los conocimientos.

Dada su etimología, la educación se connota de dos maneras: como un proceso de crecimiento estimulado desde fuera y como encauzamiento de facultades que existen en el sujeto que se educa. En la primera parte se fundamenta el concepto tradicional de educación, de corte intelectualista, donde el educador domina, sobre el educando pasivo, el acto de educar. La segunda se orienta a la educación nueva, permanente, basada en autoactividad, el autodesarrollo y la autorrealización del educando (Mialaret, citado en Besabe, 2007).

Como un concepto general se puede expresar que la educación es la formación del hombre a partir de las influencias exteriores a que es sometido y además por la virtud de su voluntad de autodesarrollo. En este sentido, la educación se divide en formal, no formal e informal (Besabe, 2007).

El mismo Besabe (2007) argumenta que la educación formal se entiende como un sistema educativo jerárquicamente estructurado, distribuido en grados y niveles, que van desde la escuela primaria hasta la universidad, incluyendo, además de estudios académicos generales, una variedad de programas especializados e instituciones para su entrenamiento profesional y técnico, de donde se desprende que se puede connotar a este proceso como los albores de la educación continua.

La educación no formal se compone de las actividades de aprendizaje que se realizan fuera del sistema educativo formalmente organizado, pero de manera estructurada y sistemática. La educación informal es definida como el proceso en el que cada individuo adquiere actitudes, valores, destrezas y conocimientos, extraídos de las experiencias diarias, los recursos e influencias de su ambiente (familia, vecinos, trabajo, juego, bibliotecas, entre otros) (Besabe, 2007).

\section{El uso de las TIC en la educación}

En el esquema 1 se aprecia la interacción de alumnos y docentes con las TIC y su convergencia en el proceso enseñanza aprendizaje. En un círculo virtuoso, los alumnos son inducidos por los docentes a la utilización de dichas herramientas (empleadas por ellos mismos); esta acción facilita la transferencia de saberes de los programas formativos facilitando el 
proceso educativo, el cual a la postre es evaluado por los mismos docentes. En este caso, la benevolencia de dicho ambiente es que permite llevarlo a cabo de forma presencial y/o virtual; esto es, en un espacio que privilegie la libertad del estudiante de interactuar a su ritmo en espacio y tiempo, maximizando su independencia con respecto a la estructura formal del espacio áulico.

Hoy se vive en una economía basada más en el conocimiento que en la materia prima y la fuerza física. En esta nueva sociedad, el intercambio de información es una actividad en ascenso y la educación es una de las áreas que bajo el influjo tecnológico ve modificados sus parámetros habituales. Ante este novedoso panorama que plantean las TIC, una pregunta que se hacen constantemente los educadores es ¿cómo se verá modificado su trabajo en una sociedad que cada vez desarrolla más actividades en el mundo on line? (Lozano y Burgos, 2007).

La educación a distancia tiene cada vez mayor aceptación por parte de las instituciones universitarias; en ésta se utiliza internet y las tecnologías multimedia para ofrecer una amplia variedad de cursos de grado y extracurriculares a los estudiantes de todo el mundo. Gracias a ellas un estudiante puede utilizar su computadora para obtener un título, aunque viva alejado de los centros educativos o carezca de tiempo para acudir a una universidad tradicional, esto es especialmente útil en el caso de la capacitación continua, imprescindible para los profesionistas.

De este modo, cambiarán los procesos actuales de enseñanza; el esquema clásico de profesor-alumno se transformará en uno donde los estudiantes tienen acceso al conocimiento a través de una variedad de medios, sin que influya el lugar donde se encuentren y puedan tomarse el tiempo necesario para aprender. El principio fundamental es que la transmisión de conocimientos por medio de la repetición, utilizado por los programas académicos tradicionales, actualmente ya es sustituida por el empleo de herramientas informáticas (USC-PSICOM, 2010).

\section{Esquema 1. Interacción TIC-comunidad académica-proceso enseñanza y aprendizaje}

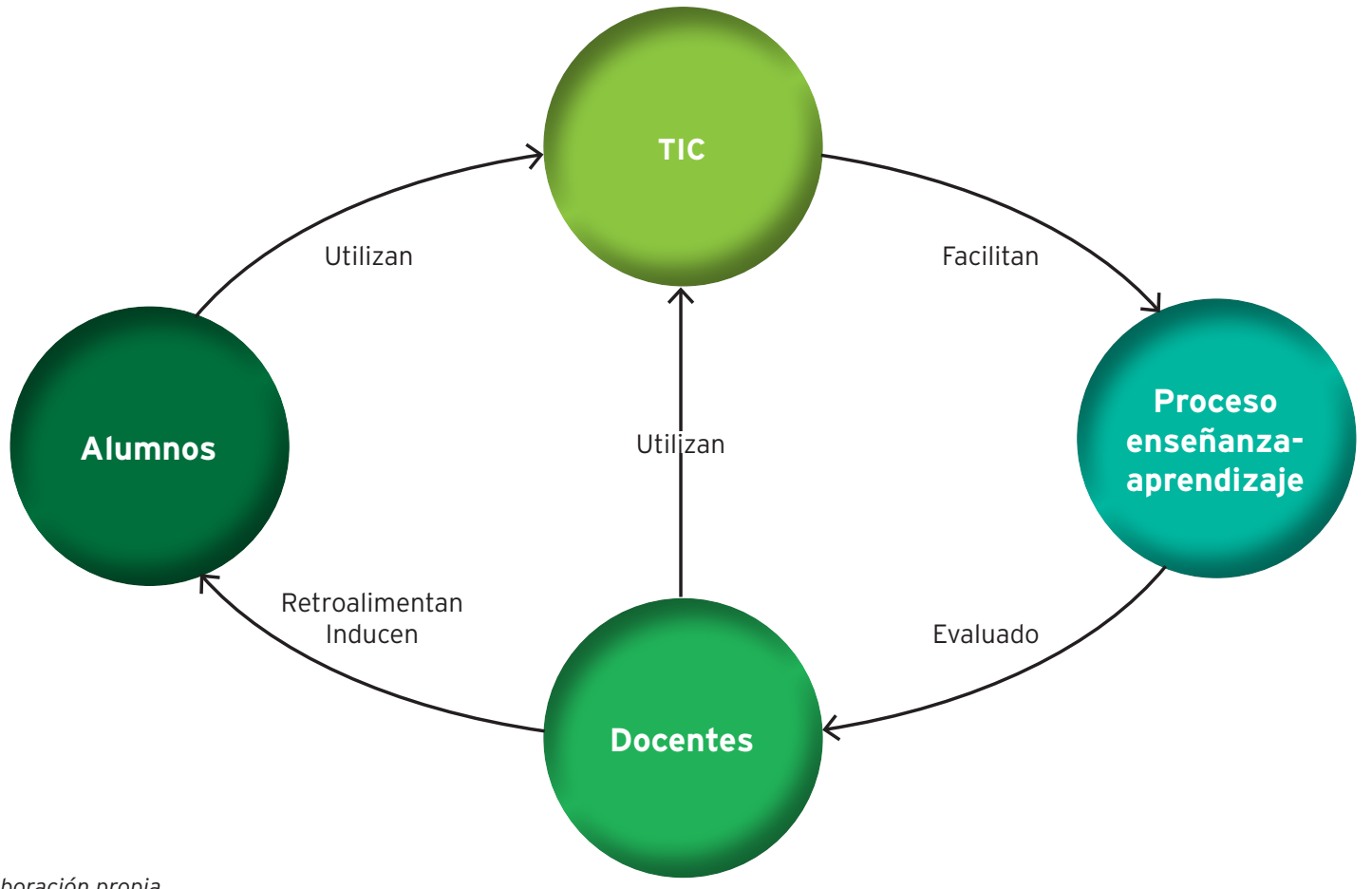




\section{Método}

\section{Unidad de análisis y muestra}

El estudio tiene un enfoque cuantitativo de corte transversal y para su desarrollo se elaboró un instrumento de acopio de información, denominado "Evaluación de la perspectiva del Modelo Educativo UANL en los jóvenes universitarios en FACPyAP", aplicado entre el 1 de octubre y el 30 de noviembre de 2014, con un total de 358 encuestados mediante muestreo aleatorio simple, de los semestres de tercero a noveno de dos de las tres licenciaturas que se ofertan en la facultad, con una edad que oscila entre los 18 y 31 años. De un universo de estudio de 2880 estudiantes, el tamaño de la muestra calculada es de 338 , con un $95 \%$ de nivel de confianza, un margen de error de $5 \%$ y una proporción de $50 \%$.

$\mathrm{El}$ instrumento de acopio contiene cinco dimensiones que amparan una estructura de 107 ítems; donde la mayoría de éstos fueron diseñados con escala de tipo Likert en niveles de (1-4), (1-5) y en algunos casos de respuesta dicotómica y de opción múltiple para mayor comprensión e interpretación del fenómeno.

En el tema de la representatividad, se logró una muestra de 245 encuestas aplicadas a mujeres, lo que constituye el $68.4 \%$; en el caso de los hombres se aplicaron 113, para un $31.6 \%$ de presencia del género masculino; esto arroja un total de 358 instrumentos válidos. En el tema de la edad se obtuvo un total de 201 encuestas para jóvenes entre 18-20 (56.1\%), 139 encuestas para jóvenes entre 21-23 (38.8\%) y 18 encuestas para jóvenes entre 24-31 años (5\%). Como conclusión, cabe hacer notar que el 95\% de las encuestas, se realizó a jóvenes con edades que oscilan entre 18 y 23 años.

De acuerdo con el programa educativo de licenciatura al que pertenecen los alumnos encuestados, 328 corresponden a la licenciatura de Relaciones Internacionales $(91.6 \%)$ y 30 de los encuestados pertenecen a la licenciatura de Ciencias Políticas $(8.4 \%)$. Se destaca el hecho de que la licenciatura de Administración y Desarrollo Sustentable es de reciente creación y su población es mínima, por lo que no se consideró en la aplicación del instrumento.

\section{Dimensiones}

El diseño del instrumento completo examina cinco dimensiones; en este artículo se contemplan tres (dimensiones 2,3 y 4 , ver tabla 1 ).

\section{Tabla 1. Dimensiones}

\begin{tabular}{|c|c|}
\hline Dimensión & Consideraciones \\
\hline $\begin{array}{l}\text { 2. Percepción de la comunidad estudiantil en } \\
\text { cuanto a la presencia de TIC en nuestra facultad }\end{array}$ & $\begin{array}{l}\text { - ¿Las TIC, son suficientes, son adecuadas? } \\
\text { - ¿EI proceso enseñanza-aprendizaje incentiva la utilización de TIC? } \\
\text { - ¿EI uso de las TIC proporcionan capacidades laborales? }\end{array}$ \\
\hline $\begin{array}{l}\text { 3. Percepción de la comunidad estudiantil en } \\
\text { cuanto al tipo y cantidad de interacción con las TIC } \\
\text { del medio ambiente }\end{array}$ & $\begin{array}{l}\text { - Utilización de medios informativos } \\
\text { - Utilización de medios electrónicos } \\
\text { - Tiempo de uso }\end{array}$ \\
\hline $\begin{array}{l}\text { 4. Percepción de la comunidad estudiantil en } \\
\text { cuanto a la utilización de TIC en nuestra facultad }\end{array}$ & $\begin{array}{l}\text { - ¿Cuál es el nivel de utilización de las TIC por alumnos? } \\
\text { - ¿Cuál es el nivel de utilización de las TIC por docentes? }\end{array}$ \\
\hline
\end{tabular}

Fuente: elaboración propia. 


\section{Resultados}

\section{Dimensión 2}

Para el caso de la apreciación de los jóvenes en la cantidad de infraestructura tecnológica existente en su facultad, en la gráfica 1 se muestra que la categoría de "suficiente-bastante" es de $34 \%$ para las mujeres y de $37 \%$ para los hombres; los alumnos refieren que dicha cantidad de infraestructura es adecuada, en contraste con el 31\% de mujeres y $30 \%$ de hombres en la categoría de "poco-nada", es decir, argumentan que es poca o insuficiente. Se observa poca diferencia con respecto al género.

En la gráfica 2, referente a la percepción que tienen los alumnos de que las materias que cursan en sus programas educativos, les permiten adquirir conocimientos y habilidades de TIC, se observa que en la categoría de "suficiente-bastante" se ubica el 37\% de mujeres y el 35\% de los hombres; en contraste, en la categoría de "poco-nada" se ubican el 31\% de las mujeres y el $23 \%$ de los hombres. De igual manera que en la gráfica 1 , se observa poca variación entre géneros.

Para el caso de la apreciación de los jóvenes en las ventajas laborales que le proporciona el manejar TIC durante su estancia académica en la facultad, en la gráfica 3 se muestra que el $50 \%$ de las mujeres y el $49 \%$ de los hombres se ubican en la categoría de "suficiente-bastante", es decir, para ambos sexos dicha actividad les favorece en gran medida. En contraste, en la categoría de "poco-nada" sólo está el 9\% de mujeres y el 10\% de hombres, para quienes dicha actividad no representa ningún beneficio. Se observa nuevamente que existe similitud entre géneros.

\section{Gráfica 1. ¿TIC suficientes y adecuadas?}

\section{¿Considera que es suficiente la cantidad de infraestructura tecnológica en la facultad?}

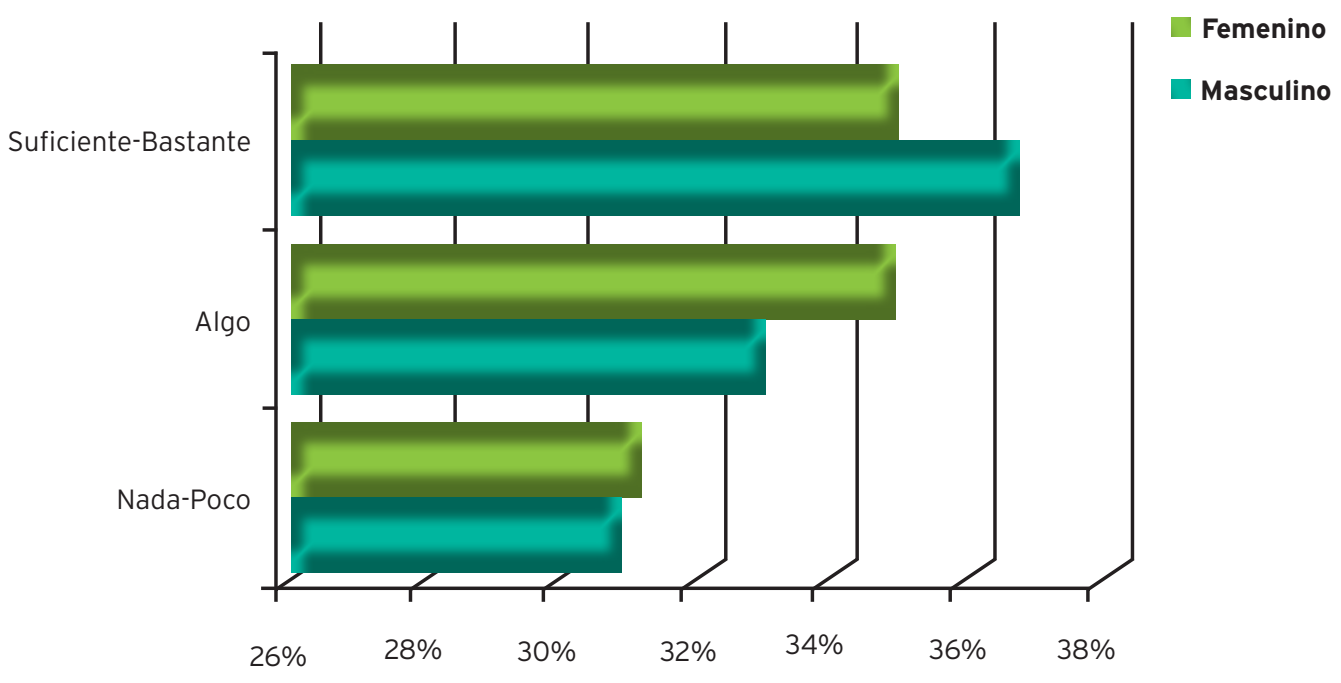

Fuente: elaboración propia. 


\section{Gráfica 2. Habilidades de TIC}

¿En que medida considera que las unidades de aprendizaje/materias le proporcionan habilidades de TIC?

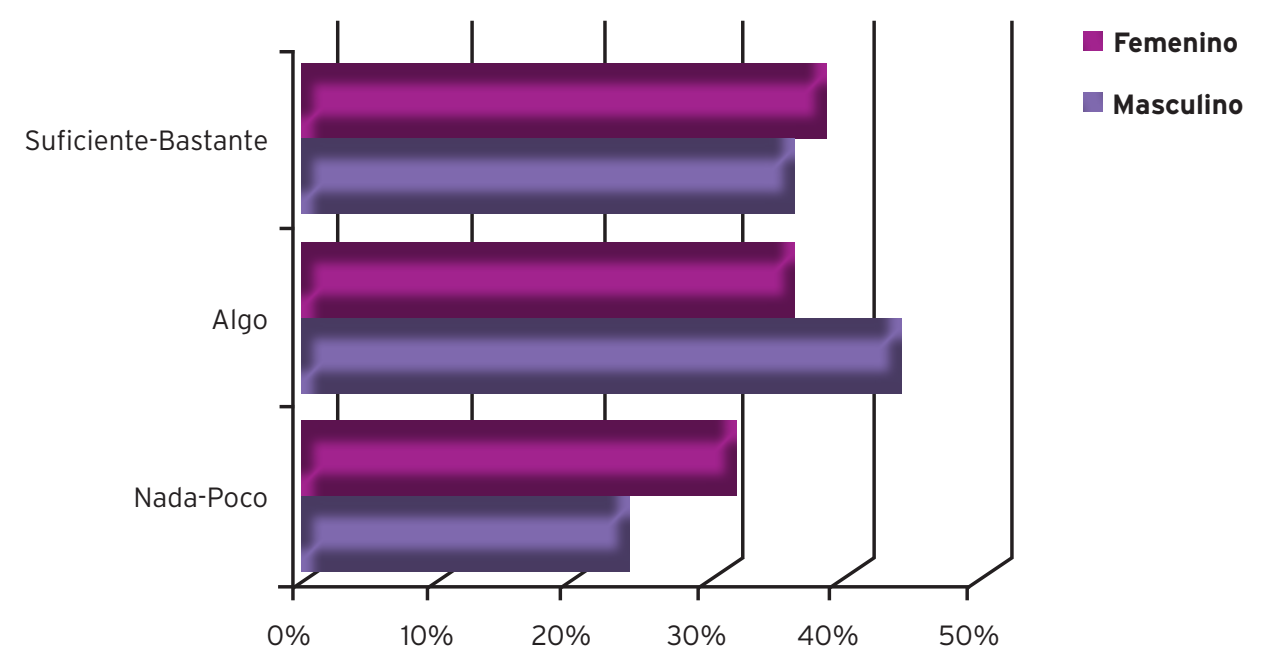

Fuente: elaboración propia.

\section{Gráfica 3. Ventajas laborales}

¿En que medida considera que el manejo de TIC le proporciona ventajas laborales?

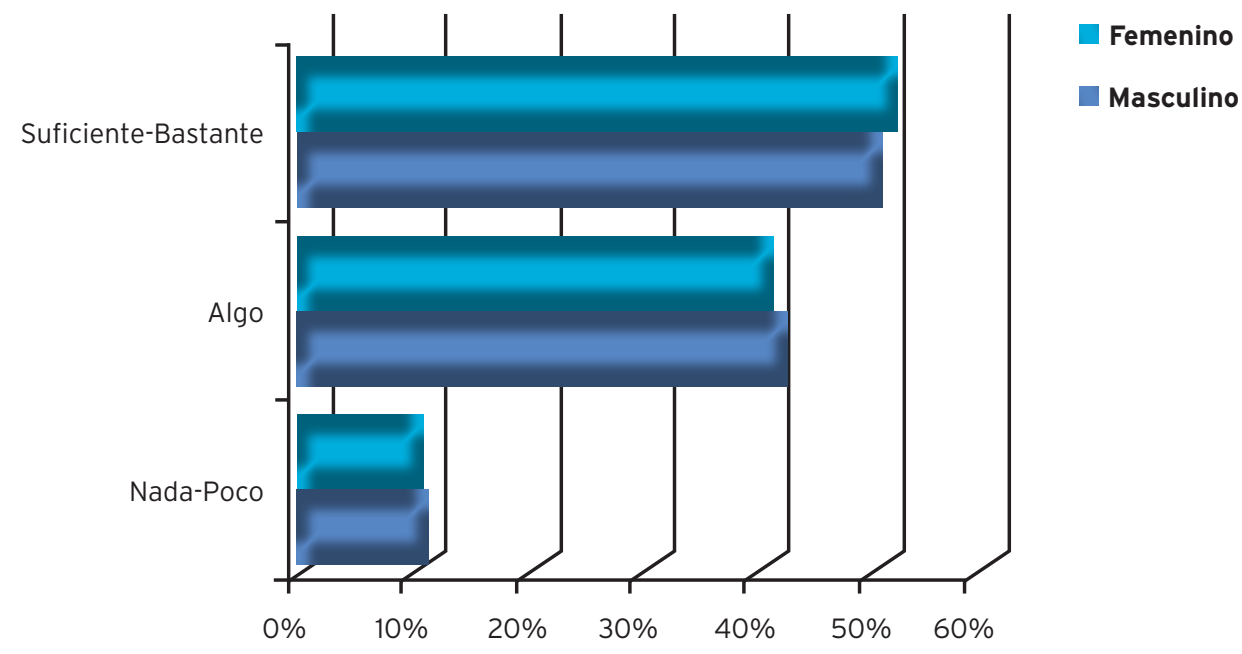




\section{Dimensión 3}

En la gráfica 4 se muestra que, para el caso de los medios tradicionales, en la categoría de "suficientebastante", los estudiantes refieren utilizar en mayor medida la televisión como medio informativo, con un $57 \%$, seguido de la prensa, con $26 \%$, la radio, con $24 \%$, y las revistas, con $19 \%$.

Con respecto al tiempo que le dedican a estos medios, en la tabla 2 se observa que se privilegia la utilización de la televisión con un promedio de 180 minutos por día, en contraste con las revistas con tan solo 43 minutos por día.

En la gráfica 5 se muestra que, en el caso de los medios electrónicos, para la categoría de "suficiente-bastante", los estudiantes refieren utilizar en mayor medida las páginas web de internet como medio informativo y de búsqueda de información, con un $96 \%$, seguido por la utilización de las redes sociales de Facebook con un $84 \%$; en contraste, en la misma categoría, sólo el 15\% dice utilizar TV por internet, y el 13\% radio por internet.

Con respecto al tiempo que dedican a estos medios electrónicos, en la tabla 3 se observa que el mayor tiempo se invierte en Facebook con 259 minutos promedio por día, seguido de Twitter con 119 minutos promedio, mientras que sólo dedican 35 minutos promedio por día a la radio vía internet.

\section{Gráfica 4. Medios tradicionales}

\section{¿En que medida considera que el manejo de TIC le proporciona ventajas laborales?}

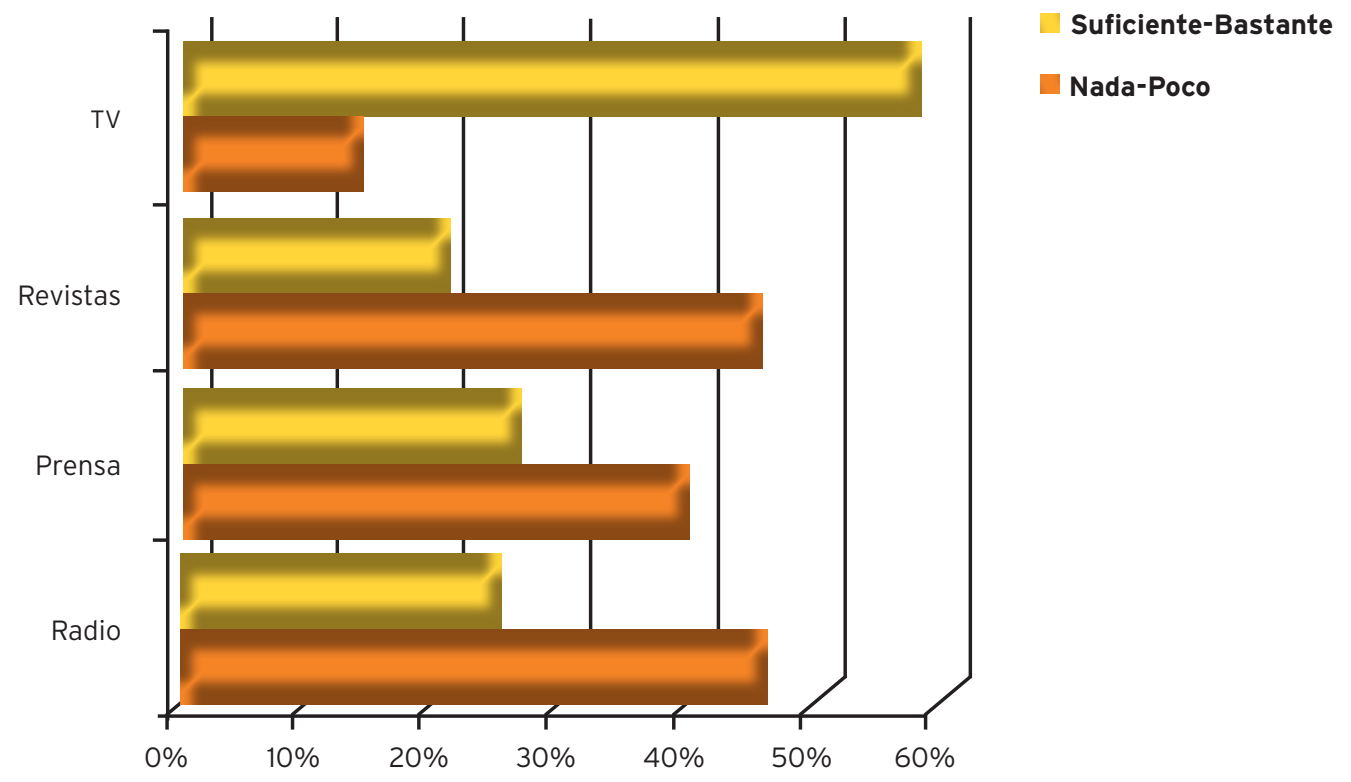

Fuente: elaboración propia. 
Tabla 2. Promedio de minutos dedicados a los medios tradicionales

\begin{tabular}{|c|c|c|c|c|}
\cline { 2 - 5 } \multicolumn{1}{c|}{} & Radio & Prensa & Revistas & TV \\
\hline Minutos & 80 & 45 & 43 & 180 \\
\hline
\end{tabular}

Fuente: elaboración propia.

\section{Gráfica 5. Utilización de los medios informativos electrónicos}

\section{Grado de utilización de medios electrónicos}

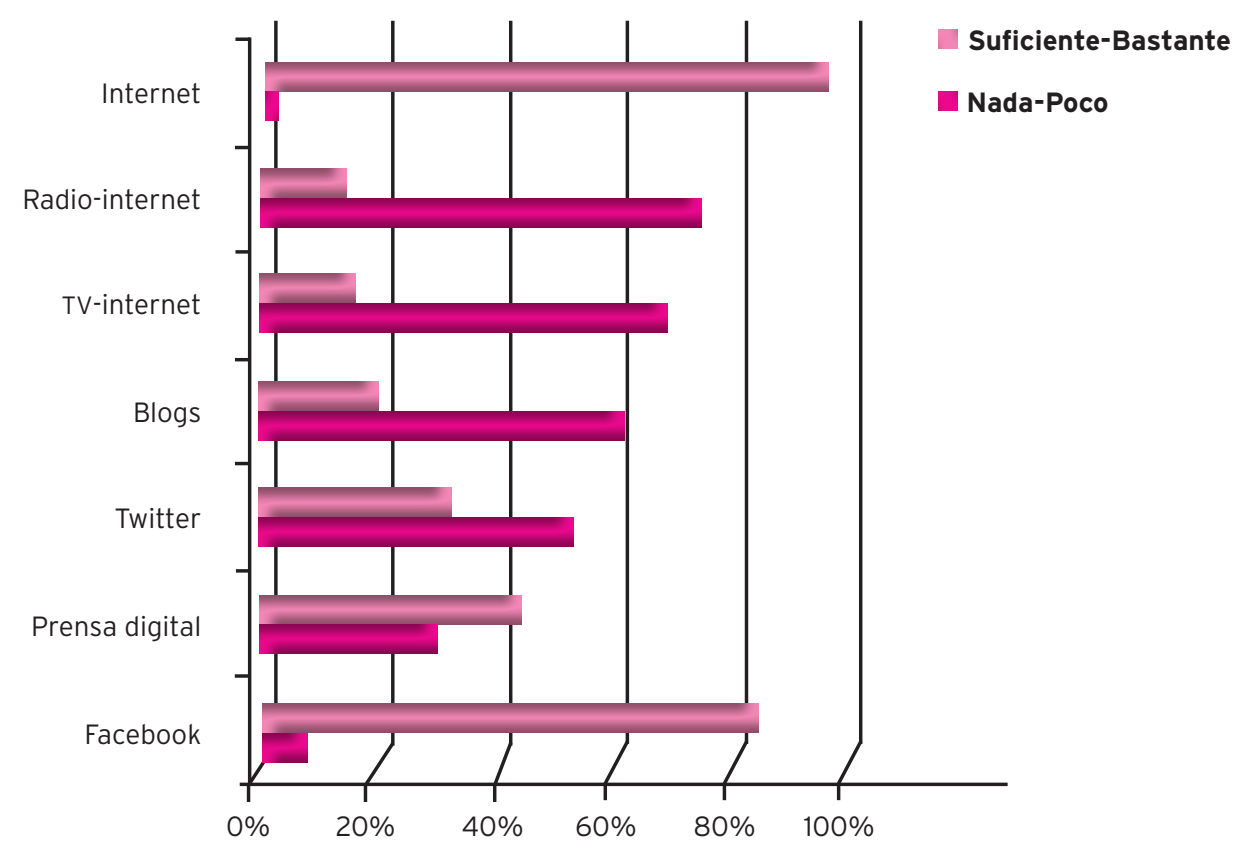

Fuente: elaboración propia.

Tabla 3. Promedio de minutos dedicados a los medios electrónicos

\begin{tabular}{|c|c|c|c|c|c|c|c|}
\hline Medio & Facebook & Prensa digital & Twitter & Blogs & TV-internet & Portales & Radio-TV \\
\hline Minutos & 259 & 70 & 119 & 38 & 38 & 70 & 35 \\
\hline
\end{tabular}




\section{Dimensión 4}

En cuanto a la apreciación de los jóvenes en el nivel de manejo de TIC durante sus actividades académicas, en la gráfica 6 se observa que, en la categoría de "suficiente-bastante", el 56\% de las mujeres y el $49 \%$ de varones refieren utilizarlas en gran medida, en contraste con el 19\% de las mujeres y el $21 \%$ de los hombres que argumentan que las utilizan "poco o nada". De nueva cuenta, se observa que existe similitud entre hombres y mujeres al respecto.

En el caso del uso de las TIC por parte de los docentes, la gráfica 7 muestra que en la categoría de "suficiente-bastante" se encuentran el $57 \%$ de las mujeres y el $52 \%$ de los hombres, mientras que sólo el 15\% de las mujeres y el 19\% de los hombres consideran que los profesores incorporan "poco-nada" las TIC en sus clases.

\section{Gráfica 6. Utilización de TIC en clases}

\section{¿Cuál es su nivel de utilización de las tecnologías de información en las actividades académicas〉}

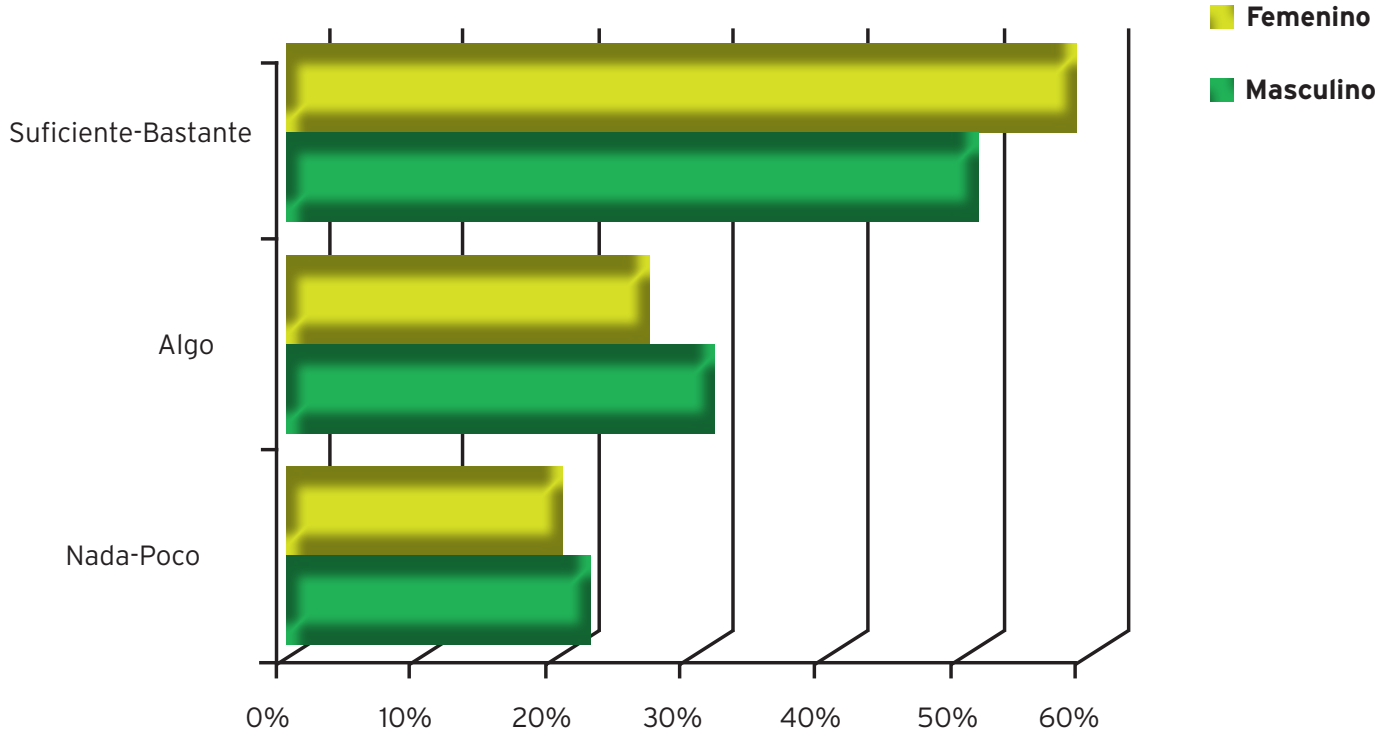

Fuente: elaboración propia. 


\section{Gráfica 7. Utilización de TIC de los docentes en clases}

\section{Los progesores incorporan el uso de las nuevas tecnologías en sus clases}

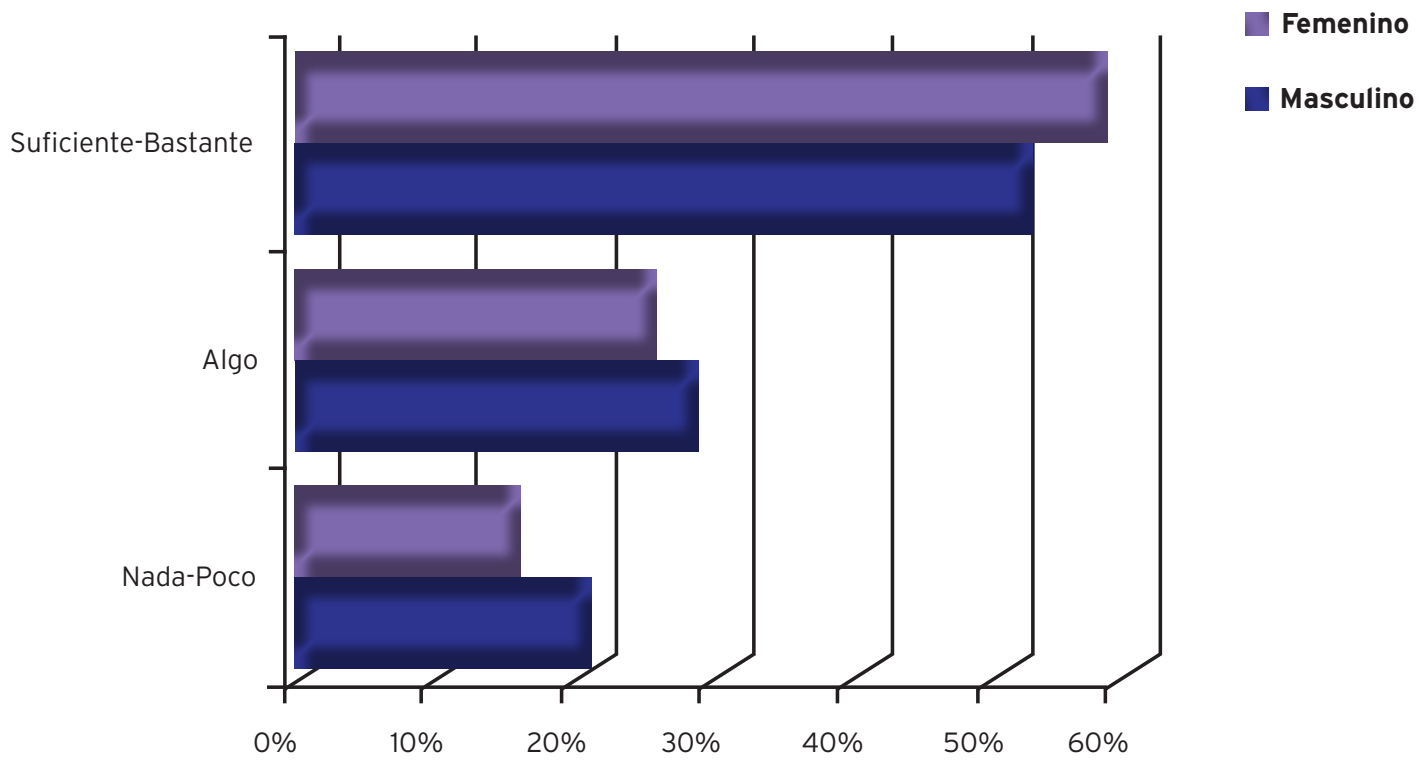

Fuente: elaboración propia.

Tabla 4. ¿Cómo considera usted que se encuentran las condiciones de las TIC en nuestra facultad?

\begin{tabular}{|c|c|c|c|c|c|}
\hline Carrera & Nada bien & Poco & Algo & Suficiente & Bastante bien \\
\hline Etapa Básica & $3 \%$ & $7 \%$ & $12 \%$ & $19 \%$ & $59 \%$ \\
\hline Licenciatura en Relaciones Internacionales (LRI) & $3 \%$ & $11 \%$ & $14 \%$ & $27 \%$ & $45 \%$ \\
\hline Ciencia Política (CP) & $4 \%$ & $12 \%$ & $18 \%$ & $23 \%$ & $43 \%$ \\
\hline
\end{tabular}

Fuente: elaboración propia.

Tabla 5. ¿Cómo considera usted que se desempeñan las TIC en nuestra facultad?

\begin{tabular}{|l|c|c|c|c|c|}
\hline \multicolumn{1}{|c|}{ Carrera } & Nada bien & Poco & Algo & Suficiente & Bastante bien \\
\hline Etapa Básica & $2 \%$ & $6 \%$ & $16 \%$ & $19 \%$ & $57 \%$ \\
\hline Licenciatura en Relaciones Internacionales (LRI) & $3 \%$ & $14 \%$ & $19 \%$ & $23 \%$ & $41 \%$ \\
\hline Ciencia Política (CP) & $5 \%$ & $17 \%$ & $18 \%$ & $21 \%$ & $39 \%$ \\
\hline
\end{tabular}

Fuente: elaboración propia. 


\section{Equipamiento Tecnológico}

En la tabla 6 se muestra la matrícula por semestre y en la Tabla 7, la cantidad y tipo de equipamiento con que cuenta la institución.

Con los datos de las tablas 6 y 7 , si se considera un universo de estudiantes de 3851 y 309 equipos de cómputo, se tendría un equipo por cada 12 alumnos, aproximadamente; ésta es una buena posición, considerando el parámetro que definió la OEI (2010), de un equipo por cada 40 alumnos para el año 2015. Esta medida ha quedado un tanto rebasada y es poco adecuada, ya que actualmente no se puede establecer la cobertura con la cantidad de equipos administrados directamente por las entidades educativas en sus espacios áulicos, debido a que en un concepto más amplio se debe tener en cuenta el acceso que tienen los alumnos a otros dispositivos personales de conexión tales como laptops, teléfonos celulares, asistentes digitales y tabletas, entre otros.

Tabla 6. Matrícula del semestre agosto-diciembre 2014

\begin{tabular}{|l|c|}
\hline \multicolumn{2}{|c|}{ Alumnos } \\
\hline Licenciatura & 3580 \\
\hline Maestría & 213 \\
\hline Doctorado y Especialidad & 58 \\
\hline Total & 3851 \\
\hline
\end{tabular}

Fuente: elaboración propia con datos obtenidos de la Secretaría de Desarrollo Institucional e Información Estratégica de la Facultad, septiembre de 2014.

Tabla 7. Cantidad de equipo instalado agosto-diciembre 2014

\begin{tabular}{|l|c|}
\hline \multicolumn{1}{|c|}{ Elemento } & Cantidad Ago-Dic. 2014 \\
\hline Equipo de Cómputo & 309 \\
\hline Video proyectores & 55 \\
\hline Pizarrones electrónicos & 51 \\
\hline Impresoras & 52 \\
\hline Teléfonos IP & 53 \\
\hline Antenas red inalámbrica & 13 \\
\hline Equipo de video conferencia & 3 \\
\hline
\end{tabular}

Fuente: elaboración propia con datos obtenidos del Departamento de Informática de la Facultad (Ago.-Dic. 2014). 
Los datos que se muestran a continuación, fueron obtenidos del Departamento de Informática de la propia facultad en el mes de septiembre de 2014. A nivel institucional, a través de la Dirección General de Informática (DGI) se provee del software estándar para el manejo de las computadoras de la UANL, en todas sus dependencias y en algunos casos provee software especializado. Así mismo, se han desarrollado diferentes programas de apoyo a las funciones docentes y administrativas, tales como el Sistema Integral para la Administración de Servicios Educativos (SIASE, con módulos de Admisión, Escolar, Finanzas y Recursos Humanos).

Los 148 profesores que pertenecen a la FCPyAP, cuentan con un espacio definido y adecuado para cubrir sus actividades de gabinete y atención de estudiantes, el cual está dotado de 13 equipos de cómputo, un video proyector, un pizarrón electrónico, equipo de sonido. Los equipos tienen la paquetería necesaria autorizada por parte de la DGI (plataforma Windows o Mac y software ofimático como Office, antivirus institucional, y suite Corel); cabe destacar el acceso a la red cableada e inalámbrica de que disponen para sus consultas, búsquedas, comunicaciones y demás actividades desarrolladas en la web. Para el caso de los profesores que además de sus labores docentes realizan tareas administrativas, se les dota de equipos de cómputo en su espacio de trabajo.

$\mathrm{El}$ acceso a la web se administra por medio de una red cableada en las instalaciones, la cual cuenta con dos troncales de conexión a los servidores de rectoría (DGI) mediante fibra óptica con una velocidad de acceso de $100 \mathrm{MB}$; los alumnos y maestros pueden hacer uso de ella a través de las computadoras que se encuentran interconectadas en dicha red.

Con este ancho de banda, se atiende tanto al servicio alámbrico como inalámbrico, y se considera suficiente ya que la población académica y estudiantil ronda las 3900 personas. Esta cantidad se divide en tres turnos, que son un aproximado de 1300 usuarios, éstos no necesariamente realizan una conexión simultánea y se supone un $80 \%$ de uso.
La red inalámbrica de la UANL, conocida como wuanl, ofrece conexión de internet en sus puntos de acceso ubicados en las diferentes dependencias universitarias, además cuenta con roaming, es decir, permite la movilización entre las diferentes áreas donde se distribuye este servicio, logrando con ello brindar un apoyo suficiente, disponible y accesible para estudiantes y docentes que posean una cuenta en el Sistema Institucional de Administración de Sistemas Escolares (SIASE).

La infraestructura inalámbrica cuenta con un total de 13 antenas (access point), con un alcance de radio de $100 \mathrm{~m}$ cada una. Si consideramos una extensión de terreno aproximada de $700 \mathrm{~m}^{2}$ en los que se ubica la institución, se tiene un promedio de $55 \mathrm{~m}^{2}$ a cubrir por cada antena, lo que se considera suficiente, ya que cada antena en promedio cubre una extensión de $280 \mathrm{~m}^{2}$ aproximadamente $\left(3600 \mathrm{~m}^{2}\right.$ en total por las 13 antenas).

\section{Discusión y conclusiones}

De acuerdo con los datos observados en el análisis, cabe destacar el hecho de que las actividades cotidianas y de formación académica se desarrollan inmersas en un ámbito tecnológico. Actualmente, el promedio de edad de los jóvenes estudiantes es de 22 años, nacieron en los albores de los años noventa; en esa década ya se oficializaba la World Wide Web (WWW), "en 1991 aparecía en el mercado la primera Lap Top de Apple" (Regil, 2011: 94).

Así, se puede concluir que esta generación nació con la web y por ende la utilización de esta herramienta se realiza per se, esto queda de manifiesto en la utilización de los medios informativos electrónicos por parte de los jóvenes (la utilización de internet en un $96 \%$ y de Facebook en un 84\%); sumando el grado de utilización de computadoras que se presenta en la región con un $56 \%$ como promedio de uso que, contrastado con el $43 \%$ de la media nacional, posiciona a la región como una zona tecnificada (INEGI, 2010). 
Esto confirma que a nivel regional los alumnos de la Facultad de Ciencias Políticas de la UANL, cuyas edades se concentran entre 18 y 24 años, son sujetos que realizan sus actividades académicas haciendo uso de las herramientas tecnológicas presentes.

Por ende, la institución se da a la tarea de potenciar dicha dinámica de utilización, poniendo al alcance de la población estudiantil programas educativos en formatos flexibles, que son ofertados utilizando las plataformas de NEXUS y recientemente VirtUANL, las cuales dosifican materiales formativos de manera virtual, independientemente de las aplicaciones tecnológicas utilizadas en los procesos administrativos de la propia institución.

Lo anterior se establece como contexto para concebir el fenómeno que se presenta en la formación integral de los educandos en esta facultad. Desde la normativa institucional, se establecen las condiciones de educación cívica y participación ciudadana, y queda explícita la actuación que debe guardar la población estudiantil y en cuyas actividades se permea la importancia de formación como alumno y como persona socialmente responsable cuando es egresado.

La sola consideración de promover la utilización de la educación a distancia en una institución educativa, no sólo conlleva la necesidad de conocer las fortalezas y debilidades tecnológicas de la institución, es imprescindible familiarizarse con las condiciones del entorno social, y en este sentido, retomando los parámetros vertidos por el Instituto Nacional de Estadística, Geografía e Informática (INEGI, 2010), se advierte que en nuestro país el equipamiento tecnológico de los hogares se presenta en un tercio de la población aproximadamente, esto da cuenta de una sociedad en crecimiento y con habilidades en el manejo de tecnologías, lo cual permite visualizar que el uso de las mismas se da en el plano laboral, educativo y doméstico.

La misma información de INEGI (2010), indica que el número de usuarios de internet a nivel nacional, representan el $64 \%$ de los mexicanos entre las edades de 12 a 34 años, los cuales utilizan internet para actividades de comunicación en mayor medida, seguido del uso educativo.

Es diferente referirse a la cantidad de equipo necesario y óptimo; la ventaja de contar con una facultad tecnificada, no implica necesariamente que se está haciendo buen uso de dichos componentes. El asunto aquí es que no sólo se requiere del mayor equipamiento posible, si no que dicha infraestructura tecnológica se utilice de forma adecuada y consistente; la aspiración implica necesariamente que los alumnos, además de contar con un acceso apropiado, tengan la capacidad de convertirlo en conocimiento y el conocimiento, en nuevas capacidades y prácticas para la vida.

Lo anterior da pauta para entender que, ya sea dentro del currículo o en el área de formación continua, se debe de fortalecer el uso adecuado de TIC, de tal suerte que los alumnos no sólo utilicen las plataformas tecnológicas como un medio de comunicación social, sino que se concienticen que son un medio de adquisición de conocimientos.

En los resultados se observa que los alumnos perciben que, en su facultad, las TIC existen en cantidad suficiente y adecuada, que los programas educativos les permiten adquirir competencias en ese sentido, lo que redundará en una ventaja competitiva en el momento de incursionar en el ambiente laboral.

Se observa también que el medio informativo tradicional más utilizado es la televisión, con un promedio 180 minutos por día de uso. En contraste con las revistas, prensa y radio, que utilizan en menor medida.

Para el caso de los medios informativos y de búsqueda de información electrónicos, sobresale la utilización de páginas web en internet como herramienta preferida en contraste con el resto de los medios. Sin embargo, cabe notar que la aplicación más utilizada de comunicación (y redes sociales) es la de Facebook con 259 minutos al día como promedio, seguida de Twitter. 
Otro de los aspectos analizados es el manejo de TIC que tienen los alumnos durante su estancia en las clases, y lo que refieren es que sí las utilizan en gran medida al igual que los docentes.

En el tema de la percepción por carrera, se visualiza que los alumnos de la etapa básica tienen una mejor percepción de las condiciones de las TIC en la facultad, que los que pertenecen a la licenciatura de

\section{Referencias}

Besabe, Fabian (2007), Educación a distancia. En el nivel superior, México, Trillas.

Castillejo, José Luis (2007), Diccionario de las ciencias de la educación, México, Santillana/Océano.

Cobo, Juan Cristóbal (2009), "El concepto de tecnologías de la Información: Benchmarking sobre las definiciones de las TIC en la sociedad del conocimiento", en Revista de Estudios de Comunicación ZER, vol. 14, núm. 27, pp. 295-318, ISSN: 1137-1102. <http://www.ehu.eus/ojs/index.php/Zer/article/ view/2636/2182> [Consultado: noviembre de 20015].

Dussel, Inés y Luis Quevedo (2010), VI Foro Latinoamericano de Educación. Educación y nuevas tecnologías: los desafios pedagógicos ante el mundo digital, Buenos Aires, Santillana, <http://www.unsam.edu.ar/escuelas/humanidades/ actividades/latapi/docs/Dussel-Quevedo.pdf> [Consultado: octubre de 2014]

Fernández, Juan Manuel (2013), Las tecnologías de la información y la comunicación desde la perspectiva de la psicología de la educación, México, Escuela de Graduados en Educación, Instituto Tecnológico de Monterrey, <http://catedra. ruv.itesm.mx/handle/987654321/649; http://hdl. handle.net/11285/578185> [Consultado: noviembre de 2014].

Instituto Nacional de Estadística y Geografía (INEGI) (2010), México en cifras: Información por entidad federativa,
Relaciones Internacionales y los de Ciencia Política; así como la percepción de desempeño de éstas en la institución.

Con lo anterior se concluye que existen las condiciones necesarias y suficientes para implementar programas cien por ciento virtuales, lo que redundará en el cumplimiento de la flexibilización educativa de la institución en su visión 2020.

Nuevo León, México, INEGI, <http://www3.inegi. org. $\mathrm{mx} /$ sistemas/mexicocifras/default.aspx? $\mathrm{e}=19>$ [Consultado: febrero de 2015].

Lozano, Armando y Vladimir Burgos (2007), Tecnología educativa: en un modelo de educación a distancia centrado en la persona, México, Limusa.

Mena, Manuel (1996), La educación a distancia en el sector público, Buenos Aires, INAP.

OCDE (2015), Panorama de la educación en México, México, <https://www.oecd.org/mexico/Education-at-aglance-2015-Mexico-in-Spanish.pdf> [Consultado: noviembre de 2015].

Picardo, Oscar, Juan Carlos Escobar y Rolando Balmore (2005), Diccionario Enciclopédico de Ciencias de la Educación, República de El Salvador, CIE Colegio García Flamenco, <http://www.insumisos.com/ lecturasinsumisas/Diccionario $\% 20$ enciclopedico $\% 20$ de\%20Educacion.pdf> [Consultado: marzo de 2016].

Regil, Laura (2011), "Aprendizajes y TIC en Educación Superior", en Reencuentro, núm. 62, México, Universidad Autónoma Metropolitana unidad Xochimilco, pp. 92-96, <http://www.redalyc.org/articulo. oa?id=34021066011 > [Consultado: febrero de 2015].

Sarramona, Jaume (1990), Tecnología educativa: una valoración crítica, Barcelona, CEAC.

Tello, Edgar (2008), "Las tecnologías de la información y comunicaciones (TIC) y la brecha digital: su impacto 
en la sociedad de México", en Revista de Universidad y Sociedad del Conocimiento, vol. 4, núm. 2, Barcelona, Universidad Oberta de Catalunya, pp. 1-8, <http:// www.Dialnet-LasTecnologiasDeLaInformacionYCo municacionesTICYLa-2521723.pdf> [Consultado: enero de 2015].

Universidad Autónoma de Nuevo León (UANL) (2012), Plan de Desarrollo Institucional 2012-2020, México,
UANL, <http://www.uanl.mx/sites/default/files/ documentos/universidad/pdi-2020-26abril.pdf> [Consultado: febrero de 2015].

UANL (2008), Plan de Desarrollo Institucional 2007-2012, México, UANL, <http://www.uanl.mx/sites/default/ files/documentos/universidad/plan-desarrollo-20072012-v09.pdf> [Consultado: marzo de 2015].

\section{Cómo citar este artículo:}

